\title{
Horseshoe Kidney Presenting As Appendicitis
}

\author{
Dr. Snehansu Pan MS.DNB \\ Associate Professor, General Surgery Burdwan Medical College, BURDWAN
}

\begin{abstract}
Clinical diagnosis, though usually correct, sometime may be challenging. This is due to the complexity of medical science. Here is a patient, who is diagnosed as recurrent appendicitis but after routine ultrasonography, come out to be a case of horseshoe kidney. When complications occur in such a kidney; it present as some other abdominal pathology. This is because horseshoe kidney is not a commonly considered clinical condition. Textbook says it is possible, but it is difficult to consider this during routine clinical practice. Keywords: Congenital abnormality, Horseshoe kidney, Appendicitis, Diagnostic confusion.
\end{abstract}

\section{Introduction}

Appendicitis is a common surgical condition. Diagnosis is 'very easy' and can be made by any clinician. And as the treatment for any pathology of appendix is removal, some time clinicians are trapped. Patients continue to suffer. Because so many abdominal conditions mimic appendicitis. So unless proper care is taken and base line investigations are done, one may made a wrong diagnosis and treatment. Here is a patient clinically diagnosed as appendicitis but when investigated found to have complicated horseshoe kidney. This was completely unsuspected,

\section{Case presentation}

A 21 years old unmarried female patient came to our clinic with complaints of chronic right lower abdomen pain for last 2 years. She had no history of fever, nausea, vomiting, malaena or burning micturation. Her menstrual history was normal. There was no history of operation or intervention.

Examination revealed a healthy subject. Temperature was normal. Pulse 80/min. B.P.-120/80. Abdomen was soft, scaphoid. There was a localized tenderness over lower abdomen just right to the umbilicus. No lump detected. Overlying skin and umbilicus was normal. Digital rectal examination was within normal limit. A clinical diagnosis of recurrent appendicitis was made. The following routine investigations were done.

\section{Investigations:}

1. Blood: Hb-12.8\%, TC-7600, N-76\%, E-3\%, L-16\%, M-5\%, B-0\%.FBS-98 mg/ dl, Urea-19 mg/dl, Creatinin$0.75 \mathrm{mg} / \mathrm{dl}$.

2. Urine: Microscopy shows pus cells and bacteria. Culture reveals gram negative bacillus.

3. U S G: Right kidney is located slightly down below in right iliac fossa and hypoplastic. Left kidney is enlarged in size. Lower pole of both kidneys is continuous with in midline in front of aorta. Impression: Horseshoe kidney with hypoplastic ectopic right kidney.(Fig 1)

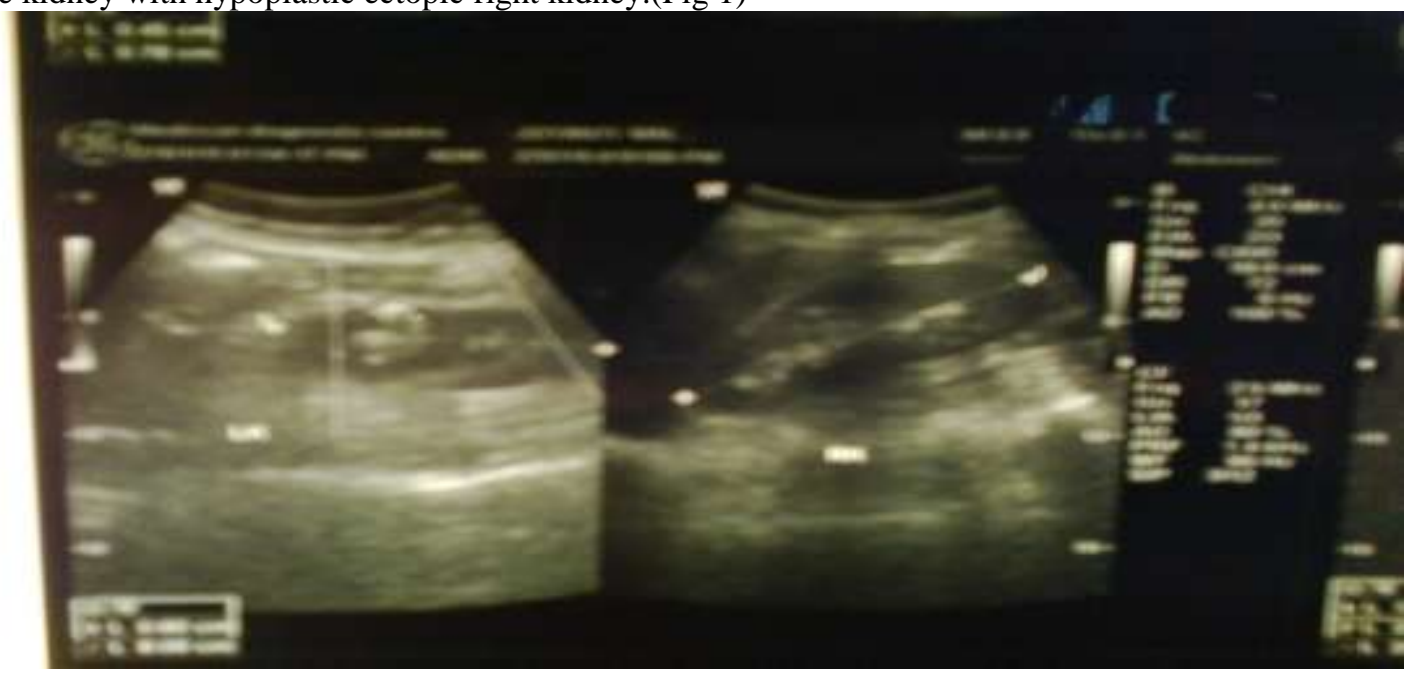

Fig 1: USG showing horseshoe kidney 
4. Intravenous urogram: Right kidney is low down in position and appears small. Rotation of left kidney is seen. Both kidneys show no hydronephrotic changes. Lower poles of both kidneys appear fuse together at the level of L2-L. (Fig: 2)

\section{Impression:}

Horseshoe kidney with hypoplastic and low down position of right kidney.

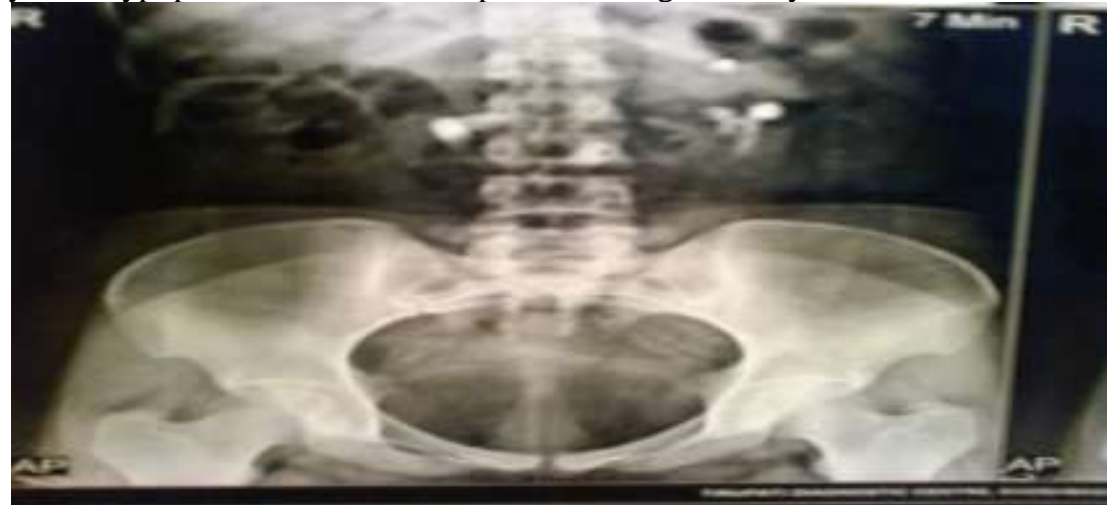

Fig 2: Intravenous Urogram showing both kidneys medially placed and calices directed anteriorly.

\section{Discussion}

Common surgical text books does not consider horseshoe kidney as a differential diagnosis of appendicitis. So this case is a unique one.

Definition: Horseshoe kidney is produced by fusion of the two metanephric masses across the midline.

Embryology: (Fig 3) The kidney systems are formed in a cranio-caudal sequence during intrauterine life in humans: the pronephros, mesonephros, and metanephros. The first of these systems is rudimentary and nonfunctional. The second may function for a short time during the early fetal period .The third forms the permanent kidney. The kidney, initially in the pelvic region, later shifts to a more cranial position in abdomen. This is called ascent of kidney. During its ascent to the abdominal level, it is vascularised by arteries that originate from the aorta at continuously higher level. The lower vessels usually degenerate, but some may remains. Sometime, the kidneys are pushed so close together during their passage through the arterial fork, that the lower poles fuse forming a horseshoe kidney. It is usually at the level of the lower lumber vertebrae, since its ascent is prevented by the root of the inferior mesenteric artery. The ureters arise from the anterior surface of the kidney pass ventral to the isthmas in a caudal direction. ${ }^{1}$

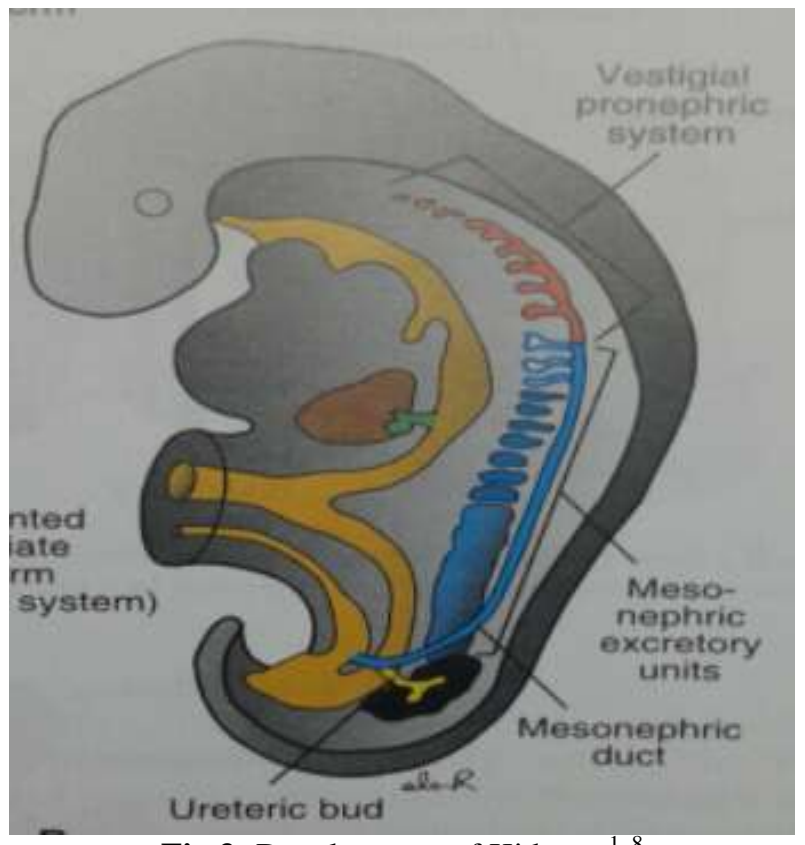

Fig 3: Development of Kidney. 
Incidence: Various authors have reported as 1 in $400,{ }^{5} 1$ in $600,{ }^{1} 1$ in $1000 . .^{2,3,4}$ It is more common in male. Fusion of the upper poles is uncommon. ${ }^{4}$

Clinical features: May be asymptomatic. Some may develop ureteral obstruction. Gastrointestinal symptoms mimicking peptic ulcer, cholelithiasis or appendicitis may be noted. Infection, hydronephrosis or calculus formation may also be noted. ${ }^{2}$ It may present as a firm mass in the pelvis. ${ }^{4,5}$ Horseshoe kidneys may be found by chance in the course of operation for aortic aneurysms. ${ }^{3}$ It may also be found during abdominal imaging for other conditions.

Associations: ${ }^{6}$ Horseshoe kidneys are frequently associated with both genitourinary and non-genitourinary malformations, and are also seen as part of a number of syndromes:

\section{Chromosomal / aneupliodic anomalies}

- Down syndrome

- $\quad$ Turner syndrome - up to $7 \%$ have a horseshoe kidney

- Edward syndrome (trisomy 18 ) - up to $20 \%$ have a horseshoe kidney

- Patau syndrome (trisomy 13)

\section{Non-aneupliodic anomalies}

- Ellis-van Creveld syndrome

- Goltz syndrome

- Kabuki syndrome

- $\quad$ Pallister-Hall syndrome

○ VACTERL association

Complications: ${ }^{2:}$ Fused kidneys are prone to ureteral obstruction because of a high incidence of aberrant renal vessels and arching of one or both ureters over the renal mass. Hydronephrosis, stone and infections are common. A large mass occupying the concavity of the sacrum may cause dystocia.

\section{Conclusion}

Logan Clendening (1884-1945), professor of clinical medicine, university of Kansas, once said "Clinical diagnosis is an art, and the mastery of an art has no end: you can always be a better diagnostician." But perhaps it has limitations, as it is evident in this case. Every clinical diagnosis should be confirmed by suitable investigations.

\section{Reference}

[1]. T W SADLER: Langman's Medical Embryology: $12^{\text {th }}$ edition (2012) p. 232-240.

[2]. Emil A Tanagho and Jack W McAninch: SMITH'S General Urology: $12^{\text {th }}$ edition (1988) p. 502-504.

[3]. John Blandy and Christopher Fowler: Urology: $2^{\text {nd }}$ edition (1996) P. $85-86$.

[4]. R C G Russel et al : Bailey \& Love's Short practice of surgery: $23^{\text {rd }}$ edition (2000) p-1174.

[5]. Herold Ellis et al: General Surgery: $12^{\text {th }}$ edition (2010), p- 335-336.

[6]. Dr Yuranga Weerakkody and Dr Frank Gaillard et al: Horseshoe kidney: Google Search, 2014.

[7]. Allan Clain: Hamilton Bailey's Demonstrations of Physical Signs in Clinical Surgery: $17^{\text {th }}$ edition (1986) p. 350. Richard S Snell: Clinical Anatomy by Region, 9th edition (2012), p-213. 